\section{CasuHAL au service de la science ouverte}

\author{
Le club des utilisateurs de l'archive ouverte HAL, CasuHAL, \\ favorise les interactions entre ses membres, et avec HAL.
}

II y a dix ans, peu auraient parié que la France se doterait d'une loi protégeant les chercheurs contre la mainmise des éditeurs scientifiques sur les publications, d'un ambitieux Plan national pour la Science ouverte (PNSO) et d'un projet européen visant le $100 \%$ de libre accès (Plan S) avec le soutien de l'agence de financement nationale ${ }^{1}$. Nous ne sommes plus à la croisée des chemins, mais bel et bien lancés sur l'autoroute de la science ouverte, et notre vitesse de croisière n'a jamais semblé aussi proche.

Dans cette perspective, $\mathrm{HAL}^{2}$ est actuellement incontournable dans le paysage académique français. L'archive ouverte fait aussi bien office de pierre angulaire par sa création en 2001 que de centre de gravité. HAL et le Centre pour une communication scientifique directe (CCSD) ont continuellement renforcé leur position au fil des conventions inter-établissements (2006 et 2013) et des plans nationaux (feuille de route des infrastructures nationales en 2016 et PNSO en 2018).

En 2019, la grande majorité des établissements de l'enseignement supérieur et de la recherche utilise HAL, soit comme leur archive ouverte officielle (près de 150 portails actifs), soit en y dupliquant le contenu de leur archive ouverte locale, comme le prévoit la convention inter-établissements.

\section{LES "PETITES MAINS" DE LA SCIENCE OUVERTE}

A l'origine, l'accès ouvert (open access) émerge grâce au principe de l'auto-archivage des publications scientifiques. Des chercheurs organisés et volontaires créent les plateformes et les procédures nécessaires à une communication scientifique directe dans leur champ disciplinaire. Les sites Arxiv (1991) et Repec (1997) en sont de parfaites illustrations. Trente ans plus tard, le paysage de la recherche a changé. Le nombre de revues, de publications et d'archives ouvertes a explosé, le contexte juridique a profondément évolué et les

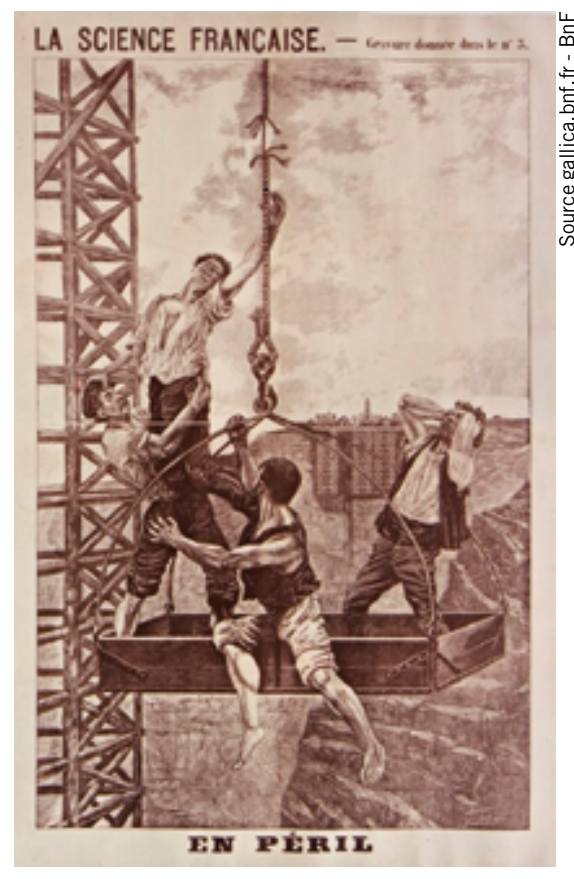

Illustration extraite de la Science française, revue populaire illustrée 1885

exigences en matière de diffusion en accès ouvert sont plus prégnantes que jamais. Aujourd'hui, derrière chaque publication déposée dans une archive ouverte, derrière chaque jeu de données diffusé librement, il y a non seulement un chercheur, mais aussi un professionnel de l'information scientifique et technique qui œuvre. Des centaines d'agents forment, conseillent et sensibilisent les chercheurs aux stratégies de diffusion en accès ouvert, créent et administrent les portails, déposent et référencent les productions scientifiques, enrichissent et nettoient les référentiels, valorisent et relaient les remarques et critiques des chercheurs sur les outils utilisés.

Ces "petites mains" de la science ouverte se sont organisées fin 2016 en réseau professionnel au sein d'une association loi 1901 : CasuHAL, club utilisateur de l'archive ouverte HAL. Les motivations à l'origine de ce regroupement sont multiples. Le besoin de briser un certain isolement arrive probablement en premier. Une étude interne

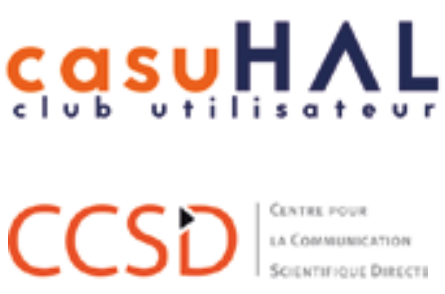

ta science partagee

réalisée fin 2017 montre que les membres de l'association travaillent le plus souvent seuls sur leur archive ouverte. Rencontrer et échanger avec des pairs constitue un réflexe professionnel indispensable face à un environnement mouvant.

\section{DES INTERACTIONS RÉGULIÈRES}

Grâce aux interactions régulières avec les chercheurs de leurs établissements et à l'administration quotidienne de leur portail ou de leur collection HAL, les membres du réseau peuvent déterminer les marges de progression ou les nouvelles fonctionnalités nécessaires à l'archive ouverte. Débattues au sein de l'association, ces propositions sont ensuite remontées et discutées avec le CCSD. L'objectif est d'influer moins isolément et plus efficacement sur les évolutions futures de la plateforme.

CasuHAL est d'abord un espace d'échange pour ses 330 adhérents, articulé autour de visioconférences attirant chaque mois entre 30 et 50 participants, d'une liste de diffusion, d'un wiki pour le partage des bonnes pratiques et d'un site web pour la promotion ${ }^{3}$. Enfin, les membres comptent porter une réflexion de fond sur l'avenir des plateformes de libre accès au moment de l'expansion mondiale de la science ouverte. Ils sont désormais bien souvent soumis à un double impératif : la diffusion ouverte de la science et l'assistance au pilotage de la production scientifique de leur établissement. Comme le montre le succès des archives ouvertes locales, ces positions sont complémentaires et non pas contradictoires. CasuHAL travaille, avec ses partenaires, à leur rapprochement.

Nicolas Alarcon

Université de La Réunion - CasuHAL nicolas.alarcon@univ-reunion.fr

Marlène Delhaye

Aix-Marseille Université - CasuHAL marlene.delhaye@univ-amu.fr

[1] Agence Nationale de la Recherche (ANR)

[2] Hyper articles en ligne : hal.archives-ouvertes.fr [3] www.casuhal.org 